\title{
marketplace
}

\section{Comfortable positioning}

Kodak Dental Systems provide Kodak quality images that help improve efficiency and allow dental professionals to concentrate on their patients. Kodak's RVG Systems provide a complete set of RINN ${ }^{\circledR}$ XCP-DS positioners, which enable precise and comfortable positioning of the sensors in the mouth, helping to ensure high quality images are captured that are free from distortion.

RINN $^{\circledR}$ XCP-DS positioners are designed for all types of examinations, including perapical, endodontic and bitewing. The RINN ${ }^{\circledR}$ Positioning System includes colour-coded bite blocks that are compatible with existing RINN ${ }^{\circledR}$ and aiming rings for silver halide films. Arms, aiming rings and sensor holders are also colour coordinated and it is possible to sterilise all positioners in an autoclave. For further information please contact Kodak Dental Systems on 0800895113 or visit www.kodak.co.uk/dental.

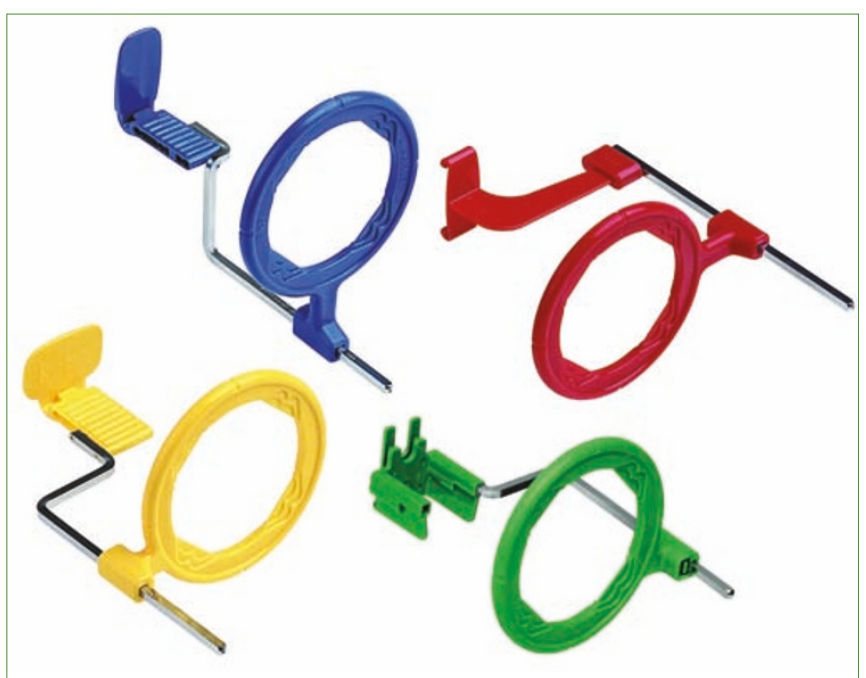

\section{Ergonomic seating solutions}

Inadequate seating can often result in back and neck pain and can have long-term detrimental physical effects. In response to such common ailments among dental practitioners, Evident now supplies DynamoSeat and InCharge saddle stools from the Dynamostol range.

Produced in Denmark, these ergonomic stools automatically correct your sitting position, enabling you to achieve perfect balance no matter what height you may be working at. Full mobility is assured by the stool's soft castors, which are suitable for all

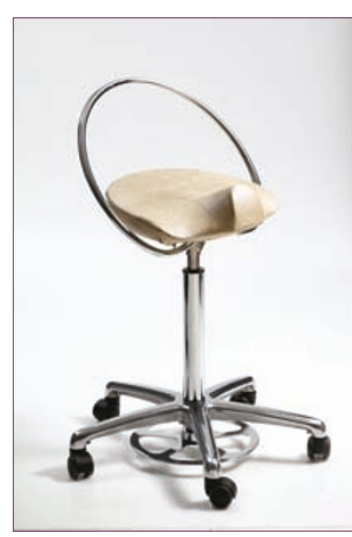

types of floor surface. Washable fabric seat covers ensure cleanliness and hygiene and the easy to replace covers mean these stools are an excellent investment.

DynamoSeat and InCharge are available in a range of differing styles and colours to complement your working conditions and the function of your practice. For a no-obligation consultation on the DynamoStol range, contact Evident on Freephone 0500321111.

\section{Better brushing in-between}

Curaprox knows that good dental health and gum care is easy to achieve and maintain using the correct techniques and a little discipline, and most importantly, the right tools. As experts in oral hygiene, Curaprox offers a superb choice of interdental brushes that have been designed to effectively clean the vulnerable interdental spaces and keep them free of plaque.

The immensely popular and autoclaveable Interdental Access Probe and Card allows you to identify to the patient the correct brush size to use in each interdental space. To compliment this

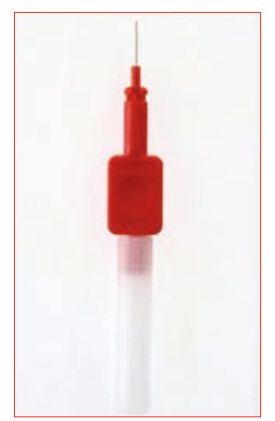
range, Curaprox is delighted to announce the introduction of a new fixed head interdental brush range, which patients will find instantly ready and easy to use.

The range is available in five colourcoded sizes: turquoise $(0.4-4.5 \mathrm{~mm})$, pink $(0.6 \mathrm{~mm})$, red $(0.5 \mathrm{~mm})$, yellow $(0.7 \mathrm{~mm})$ and green $(0.8 \mathrm{~mm})$. For more information on the new range, please contact Curaprox on 01480862084.

\section{Custom Care from Sonicare}

Sonicare has launched a new toothbrush system to elevate its family of sonic brushes to a new level.

The Sonicare Elite e9000 range has been specially designed around the individual needs of each patient. A new smaller brush head has been designed to suit children, those with smaller mouths and young people undergoing orthodontic treatment and a standard brush head is also provided so that each patient can choose the head that best suits their needs. The dual speed options - 'max' and 'gentle' - allow the brusher to customise their brushing experience and a Smartimer ${ }^{\circledR}$ feature encourages two minutes of accurate timed cleaning, helping patients keep to their dentists' recommended brushing time. A programmable Quadpacer function is also included, to encourage equal importance to be placed on brushing each quadrant of the mouth effectively.

The new e9000 is available for dental professionals and can be sold directly from your practice to the patients. For more information please contact Tracy Posner

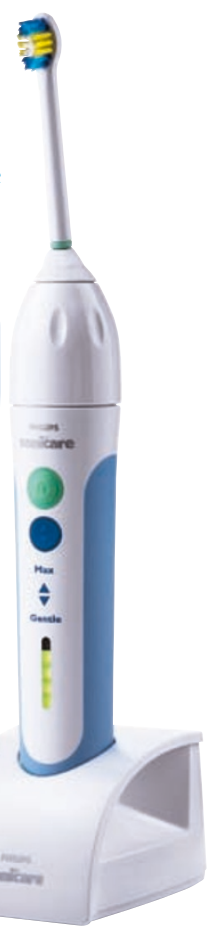
on 02085668811 or email tracyposner@btconnect.com. 